\title{
Tunable mechanical resonator with aluminum nitride piezoelectric actuation
}

\author{
J. Olivares ${ }^{*}$, J. Malo ${ }^{a}$, S. González ${ }^{a}$, E. Iborra ${ }^{a}$, I. Izpura ${ }^{a}$, M. Clement ${ }^{a}$, A. Sanz-Hervás ${ }^{a}$ \\ J.L. Sánchez-Rojas ${ }^{\mathrm{b}}$, P. Sanz ${ }^{\mathrm{b}}$ \\ ${ }^{a}$ Grupo de Microsistemas y Materiales Electrónicos, Universidad Politécnica de Madrid, \\ Madrid, Spain \\ ${ }^{\mathrm{b}}$ Dpto. de Ingeniería Eléctrica, Electrónica y Automática, Universidad de Castilla-La Mancha, \\ Ciudad Real, Spain
}

\begin{abstract}
The electromechanical response of piezoelectrically-actuated AlN micromachined bridge resonators has been characterized using laser interferometry and electrical admittance measurements. We compare the response of microbridges with different dimensions and buckling (induced by the initial residual stress of the layers). The resonance frequencies are in good agreement with numerical simulations of the electromechanical behavior of the structures. We show that it is possible to perform a rough tuning of the resonance frequencies by allowing a determined amount of builtin stress in the microbridge during its fabrication. Once the resonator is made, a DC bias added to the AC excitation signal allows to fine-tune the frequency. Our microbridges yield a tuning factor of around $88 \mathrm{~Hz} / \mathrm{V}$ for a $500 \mu \mathrm{m}$-long microbridge.
\end{abstract}

Keywords: Aluminum nitride, piezoelectric actuation, microresonator, simulation.

\section{INTRODUCTION}

Mechanical resonators are of significant interest in several application areas such as frequency filtering or gravimetric chemical sensing. High frequency stability and high quality factor are the most appreciated characteristics of these devices. Various electromechanical transduction mechanisms such as electrostatic ${ }^{1}$, magnetostrictive ${ }^{2}$, thermal ${ }^{3}$ and piezoelectric ${ }^{4}$ have been developed in the last years. Among them, capacitive and piezoelectric actuation methods are preferred because they provide a dual transduction process (i.e. the active element can be used for both detection and actuation). A great deal of effort has been expended in the capacitive transduction ${ }^{5,6}$. However, the efficiency of the electrostatic actuation and sensing decreases dramatically at high frequencies; in this case very high actuation voltages are required. Piezoelectric excitation turns out to be a good alternative to electrostatic excitation as it generally requires lower actuation voltages ${ }^{7}$; besides, the drop in the efficiency of transduction as the size of the resonators is reduced to achieve higher frequencies is less pronounced. PZT thin films have been the most frequently used materials for piezoelectric actuation ${ }^{8}$. Recently $\mathrm{ZnO}$ has been employed in AFM tips ${ }^{9}$. In the last few years, AlN has started to be considered as a good candidate for piezoelectric actuation in microsystems operating at high frequencies ${ }^{10}$; thanks to its good mechanical properties, its theoretical cutoff frequency is very high (in the $100 \mathrm{GHz}$ range) ${ }^{11}$. Besides, AlN processing is compatible with Si technologies, making AlN a very interesting material for MEMS integration.

Detection of vibration in mechanical resonators is currently carried out by the optical reflectance of a laser beam on the sample; the experimental setup requires a position sensitive detector to measure the variable deflection of the beam $^{12}$. This method, which can be applied to any kind of resonator, is frequently used in AFM systems because no artificial resonances associated to electric parasitics appear. However, for operative systems (sensors or filters) an electrical detection system is desirable. In piezoelectric actuation, the coupling of the electric fields with the strain in the material originates a significant variation of the piezoelectric capacitor admittance; this allows the detection of the

*e-mail: olivares@etsit.upm.es

MEMS, MOEMS, and Micromachining II, edited by Hakan Ürey, Ayman El-Fatatry,

Proc. of SPIE Vol. 6186, 61860K, (2006)

0277-786X/06/\$15 - doi: $10.1117 / 12.664444$

Proc. of SPIE Vol. 6186 61860K-1 
vibrational motion by electrical means, providing a very broad bandwidth along with very low power consumption. However, not all the vibrational modes are necessarily associated to admittance changes. This is, for instance, the case of a torsional mode in a perfectly symmetric structure, in which an area of the piezoelectric material is compressed whereas the adjacent symmetric area is tensioned, yielding a negligible net piezoelectric charge.

Excitation and detection of the vibrational motion in a mechanical resonator can be performed in two different ways. The first approach, consisting in a two-port system, uses separate elements for excitation and detection of the vibration $^{13,14}$. The second approach uses a single element for both excitation and detection and, thus, works as a one-port system $^{15,16}$. One-port systems using piezoelectric actuation are a good choice, as they simplify the electronic design significantly.

The shape of a mechanical resonator depends on the application it is meant for. AFM tips use typically one-sideclamped bars or cantilevers. For mass sensing or frequency filtering applications, in which higher frequencies are required, doubly-clamped beams or microbridges are more appropriate because their structure is stiffer; additionally, they provide resonance frequencies around six times higher than cantilevers of the same geometry ${ }^{12}$. In gravimetric sensor applications, the operating frequency must be as high as possible in order to increase the sensitivity. An increase of the resonance frequency for a certain vibrational mode can be achieved by reducing the mass or dimensions of the resonators. However, the decrease of the sensing area reduces the final sensitivity. A way to improve the sensitivity of the device without reducing its size is to modify the mechanical characteristics of the resonator. Previous studies demonstrate that increasing the stress of the structure ${ }^{13,17}$ produces an increase of the resonance frequency and, thus, of the sensitivity. Built-in stress induces curvature in the resonators, which increases their stiffness and, as a consequence, shifts the resonance modes to higher frequencies. Therefore, controlling the stress of the different layers of the resonator would allow to tune the resonance frequency without modifying the dimensions of the device. With this idea in mind, external tunability can be achieved by adding some mechanism capable of stiffening the whole structure. For instance, Piazza $^{13}$ uses electrostatic attraction to bend a microbridge, whereas Wang ${ }^{18}$ adds a DC voltage to the AC excitation signal in a PZT cantilever to fine-tune the resonance frequency.

In this paper we analyse the vibrational behaviour of piezoelectrically-actuated AlN microbridges for mass sensing applications. Two techniques have been used to detect the vibrational modes: one based on laser interferometry and other based on the measurement of the impedance of the actuator. Their results are compared and discussed. We also compare the vibrational modes of fully-relaxed beams with those of microbridges significantly buckled due to the effect of residual compressive stress. The variation of the resonance frequencies as a function of the initial buckling of the bridges are compared with the results of numerical simulations. Finally, we show that adding a DC bias to the AC excitation signal allows us to fine-tune the resonance frequencies within a limited range.

\section{EXPERIMENTAL}

A complete surface micromachining technology has been developed for the fabrication of microbridges. They consist of a Mo/AlN/Mo piezoelectric actuator supported by a structural layer. All these films were deposited by sputtering. Silicon dioxide $\left(\mathrm{SiO}_{2}\right)$ deposited by photo-induced CVD was used as sacrificial layer. The microbridges were easily released by removing the porous $\mathrm{SiO}_{2}$ sacrificial layer in buffered hydrofluoric acid. A more detailed description of the whole fabrication process can be found $\mathrm{in}^{19}$. We have fabricated $300 \mu \mathrm{m}$ and $500 \mu \mathrm{m}$-long microbridges. Figure 1 shows an SEM image of a $300 \mu \mathrm{m}$-long microbridge.

The different layers of the microbridge have a residual stress before the removal of the sacrificial layer. This stress depends on the sputtering conditions. When the microbridge is released, it becomes convex to partially relax the combined stress. The extra height at the centre of the microbridge with respect to the flat position is the magnitude that we will use to quantify the deformation or out-of-plane buckling of the microbridge. As resonance frequencies depend on the shape of the microbridge, we were interested in obtaining bridges with different buckling and compare their vibrational response. With that purpose, we selected the deposition conditions (substrate bias voltage and total pressure) to control the initial residual stress of the structural layer. 


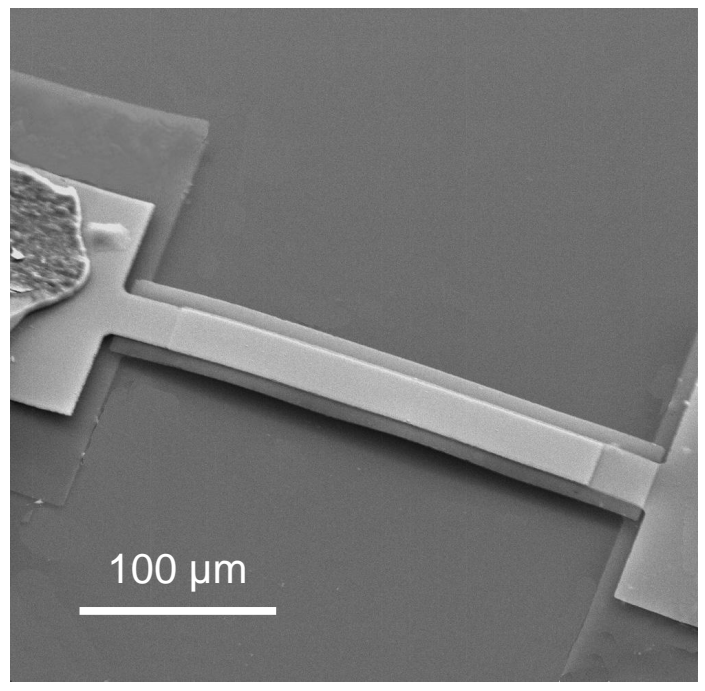

Figure 1. SEM image of a $300 \mu \mathrm{m}$-long microbridge.

To determine the residual stress, we measured the radius of curvature of the wafer before and after depositing the film; from this, the residual stress was deduced using Stoney's equation. The ranges of the stress of the different layers are indicated in table 1 together with their thickness. The out-of-plane buckling of the released microbridges was measured using a scanning electron microscope (SEM). It varied from $0.5 \mu \mathrm{m}$ to $30 \mu \mathrm{m}$ (with an uncertainty of about 0.1 $\mu \mathrm{m})$ depending on the initial stress and the length of the microbridges. Additionally, we suspect that some residual stress remains in the released microbridges, which probably affects the vibrational frequencies. Unfortunately, we cannot determine the distribution and the value of this stress.

\begin{tabular}{l|cccc}
\hline & $\begin{array}{c}\text { Structural } \\
\text { layer }\end{array}$ & $\begin{array}{c}\text { Mo bottom } \\
\text { electrode }\end{array}$ & $\begin{array}{c}\text { Piezoelectric } \\
\text { AlN }\end{array}$ & $\begin{array}{c}\text { Mo top } \\
\text { electrode }\end{array}$ \\
\hline Stress (MPa) & -500 to -3500 & 0 to -4000 & $\sim-200$ & $\sim 0$ \\
\hline Thickness (nm) & 300 & 150 & 500 & 200 \\
\hline
\end{tabular}

Table 1. Residual stress and thickness of the different layers of the microbridges.

The vibrational response of the microbridges has been measured by laser interferometry using an arrangement reminicent of the optical lever (OL) ${ }^{20}$ used in atomic force microscopy. However, in our setup, sketched in Figure 2, the photodetector is a small and fast avalanche photodiode (APD) model TIED87 from Texas Optoelectronics Inc. instead of a position sensitive detector (PSD) as in $^{20}$. The APD works as a fast detector of the time-modulated interference produced by the laser light reflected by the fixed and moving regions of the microbridges. As shown in Fig. 2, the difference between the optical paths of the two beams causes a time-dependent interference that is detected by the APD. This generates a photocurrent that is proportional to the displacement of the moving part. Due to the inherent high speed of the APD and the broad bandwidth of the detection electronics used, the system allows to measure frequencies above 2 $\mathrm{MHz}$. This technique was previously tested with one-side clamped bars or cantilevers ${ }^{21}$; it allowed us to detect the thermal motion of the cantilevers with root mean squared displacements below $10^{-10} \mathrm{~m}$. Unfortunately, the thermal motion of the doubly-clamped microbridges studied in this work is significantly below that value due to their higher stiffness. As the noise floor of the electronics lies above the thermal motion, only by applying some external driving was it possible to measure the mechanical response of the microbridges. 


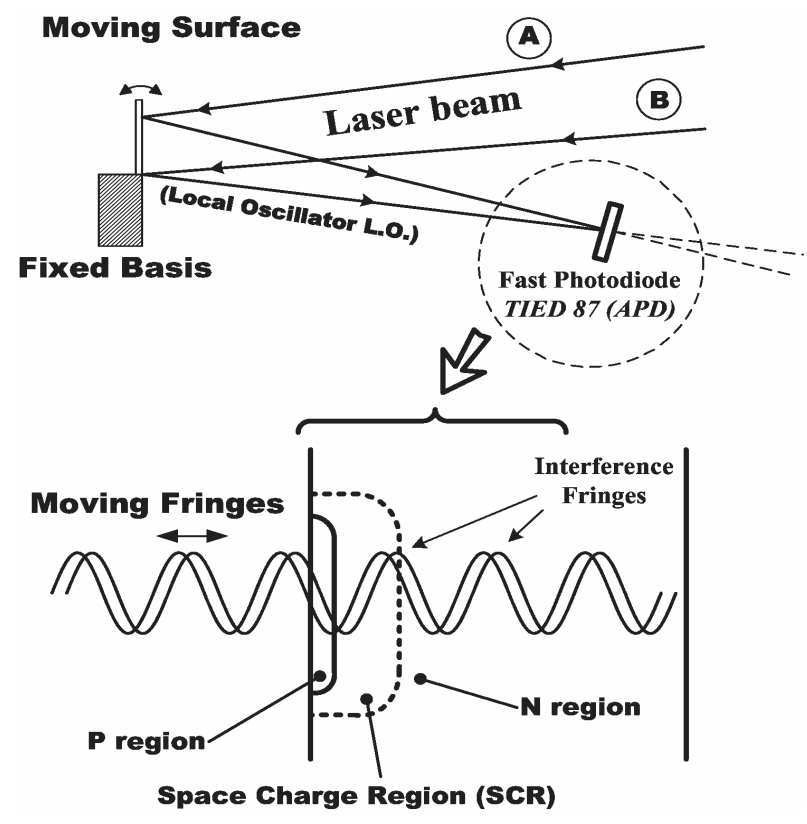

Figure 2. Laser interferometric setup used to measure the vibration of the microbridges.

The external driving is achieved simply by taking advantage of the built-in piezoelectric actuator connected to the $50 \Omega$ output of a low-power signal generator. The low output capacitance of such actuators (in the $\mathrm{pF}$ range) allows a broadband excitation of up to hundreds of $\mathrm{MHz}$, well above the few $\mathrm{MHz}$ explored in this work. Driving signals in the volt range lead to strong mechanical bending of these microbridges. Thus, the built-in tracking generator of an Agilent ESA4402 spectrum analyzer was enough to drive the microbridges. Their vibrational response was monitored by measuring the signal from the APD through a fast preamplifier $(300 \mathrm{MHz}$ bandwidth) connected to the input of the spectrum analyzer.

Due to the piezoelectric character of AlN films, their dielectric behavior depends on mechanical strain. The induced vibrations generate a modulation of the dielectric function that can be detected by measuring the admittance of the piezoelectric capacitor (Mo/AlN/Mo). We have used an Agilent 4294A impedance analyzer to measure the admittance at frequencies ranging from $100 \mathrm{kHz}$ to $1 \mathrm{MHz}$. An excitation voltage of $1 \mathrm{~V}_{\text {rms }}$ provided a good signal-tonoise ratio. All measurements were taken at $300 \mathrm{~K}$ with a relative humidity as low as $22 \%$.

Finite element analyses (FEM) were performed using CoventorWare ${ }^{22}$, a powerful software tool developed for the design, modeling and simulation of MEMS. In order to compare the experimental data with simulated results, an accurate computational model was built, as close as possible to the measured microbridges. The meshed model for the FEM analysis contains 3640 parabolic Manhattan bricks and 33269 nodes. The software solves the piezoelectric equations in the microbridge structure once appropriate boundary conditions are defined. Although in real structures the bridge supports are somewhat compliant, we have assumed that the bottom faces of the Si pillars keep a fixed position. This assumption is equivalent to a pinned or doubly-supported beam on a completely rigid substrate. The elastic, piezoelectric and dielectric constants of AIN are taken from Tsubouchi et al. ${ }^{23}$. The rest of the materials constants are taken from ${ }^{24}$.

Real structures have a large number of natural frequencies at which resonances occur, as known from classical mechanics. They can be determined by considering the dynamic response of the unexcited, unloaded structure. The solution of the differential equation of motion for a system with $n$ degrees of freedom leads to a set of $n$ eigenvalues $\lambda_{k}$, where $\lambda_{k}=\omega_{k}^{2}, k=1, \ldots, n . \omega_{k}$ is the natural frequency of the $k$-th mode of the structure. The eigenvalue calculations 
performed by CoventorWare to obtain the natural frequencies were a good but not totally realistic approximation as they did not include any damping factor, which may shift the resonance frequencies and give rise to mixed modes.

\section{RESULTS AND DISCUSSION}

\section{Effects of the residual stress of the layers}

We have analyzed the influence of buckling (thus, of the initial stress of the layers before releasing) on the frequency response of the microbridges. In figure 3 the frequency responses, obtained by reflectometry, of two $300 \mu \mathrm{m}$-long microbridges are shown. Curve (a) corresponds to a microbridge with a buckling of $0.5 \mu \mathrm{m}$; curve (b) corresponds to a microbridge with a buckling of $15 \mu \mathrm{m}$. It can be seen that both curves show a similar qualitative behavior, although as expected the frequency of the different resonance modes increases with buckling.
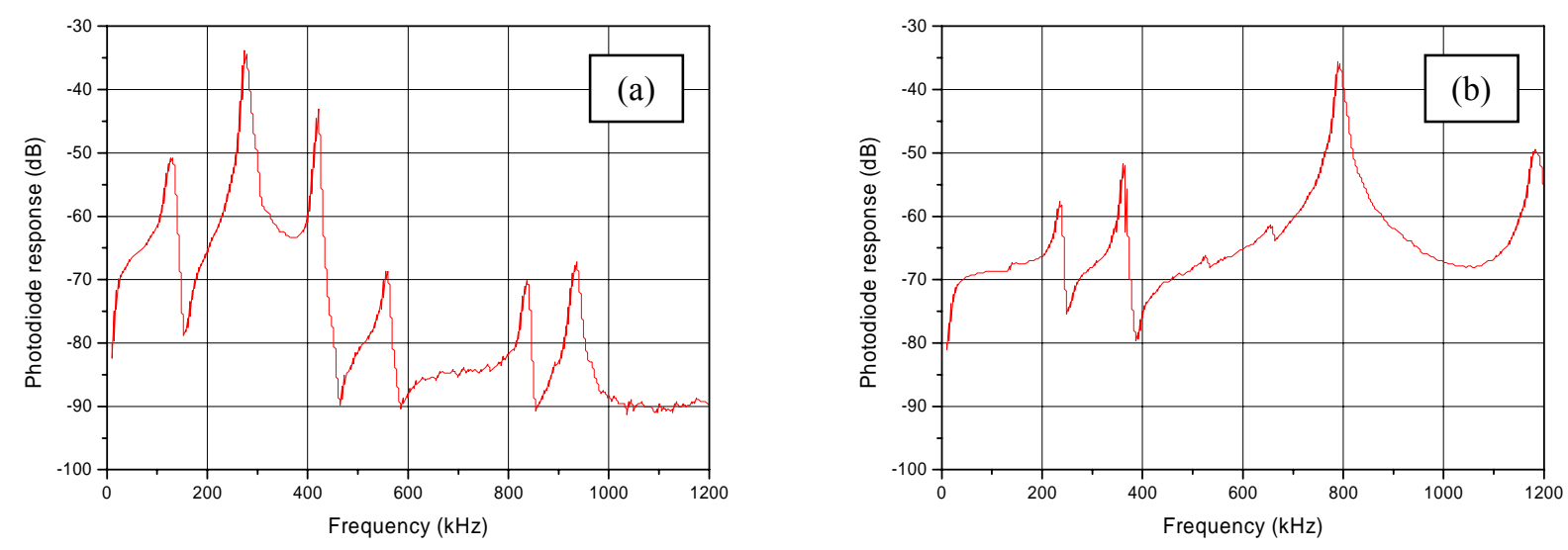

Figure 3. Frequency response of $300 \mu \mathrm{m}$-long microbridges with buckling of $0.5 \mu \mathrm{m}$ (a) and $15 \mu \mathrm{m}$ (b).

To identify the different resonance modes, we have performed CoventorWare simulations taking into account the two values of buckling, $0.5 \mu \mathrm{m}$ and $15 \mu \mathrm{m}$, considered in figure 3 . The resonance frequencies derived from the simulations are listed in table 2 together with the experimental values determined by reflectometry. Only modes below around $1 \mathrm{MHz}$ have been considered. Table 2 also includes drawings of the vibrational modes. It can be observed that the same vibrational modes appear for both kinds of microbridges. In these simulations, we have assumed that the stress of each layer is fully relaxed through buckling after removing the sacrificial layer. This is an approximation, since some stress probably remains in the actual structures.

In figure 3 modes 1, 2, 4 and 6 are flexural modes along the length of the bridge, while modes 3 and 5 are torsional modes. For the $0.5 \mu \mathrm{m}$-buckled microbridges, the simulated values of the resonance frequencies are, in general, in good agreement with the measured values. However, the frequencies of the torsional modes ( 3 and 5 ) do not agree so well. These discrepancies may be related to defects in the fabrication of the microbridges, such as asymmetries or misalignments, and also to the fact that damping and post-buckling stress are neglected. All this can induce shifts in the resonance frequencies that are not taken into account in the simulations. 


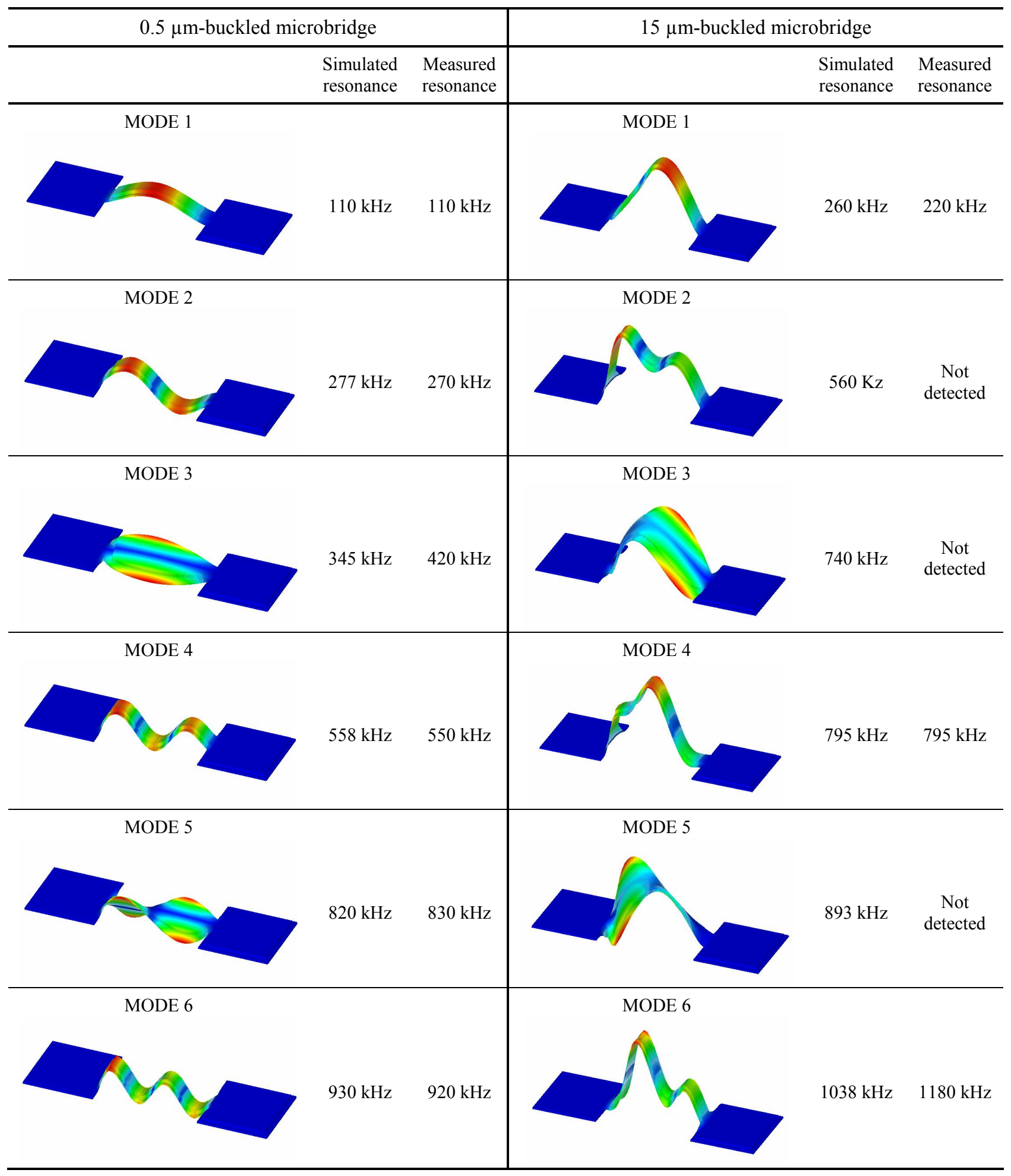

Table 2. Simulated and measured resonance frequencies for the first six resonance modes. 
In the case of the $15 \mu \mathrm{m}$-buckled microbridges, the correspondence between the measured and the simulated modes is less obvious. A peak predicted by the simulations at $560 \mathrm{kHz}$, mode 2 in table 2, does not appear in the measured spectra; however, we have observed two unidentified modes at $510 \mathrm{kHz}$ and $625 \mathrm{kHz}$. The resonance modes predicted by the simulations at $740 \mathrm{kHz}$ and $893 \mathrm{kHz}$ do not show up in the measured spectra either. A peak observed at $1180 \mathrm{kHz}$ is tentatively identified as the resonance of mode 6 , simulated at $1038 \mathrm{kHz}$. Additionally, we have measured a resonance peak at around $365 \mathrm{kHz}$ that is not predicted by the simulations. The discrepancies may be explained as follows: in all these simulations we have assumed that the stress of each layer is fully relaxed through buckling after the removal of the sacrificial layer. The discrepancy with the measured data suggests that the real structure retains part of the initial stress. Besides, the stress distribution is probably not homogeneous across the thickness or over the plane. The effect of residual stress cannot be simulated, since its magnitude and spatial distribution are not known.

Both measurements and simulations indicate that the out-of-plane buckling of the microbridges affects their resonance frequencies. Resonance frequencies increase with buckling. High frequencies are desirable in gravimetric chemical sensors, as their sensitivity improves with operating frequency. Therefore, the intentional control of the initial residual stress of the different layers of the microbridge, which determines its buckling, is a means to optimize its resonance frequencies for each specific application. This feature can be used to pre-tune the microresonators during the fabrication process.

Figure 4 shows the variation of the simulated frequencies of the six modes shown in table 2 as a function of the out-of-plane buckling. For flexural modes $(1,2,4$ and 6$)$ the resonance frequency initially increases with buckling, but then saturates at high buckling values. The two torsional modes ( 3 and 5) show a different behavior.

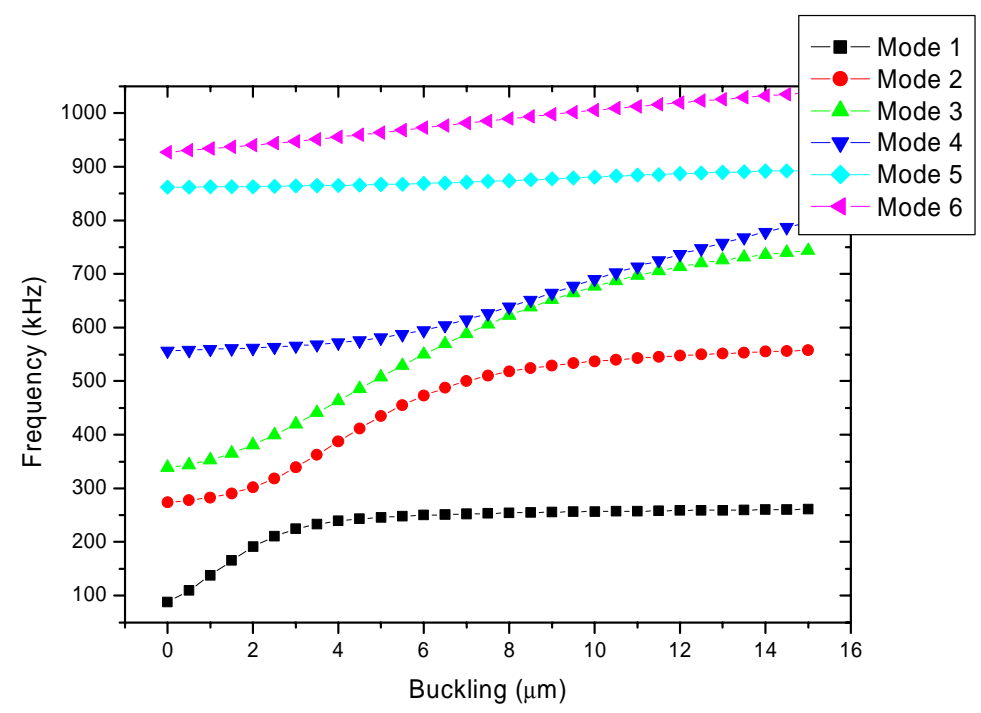

Figure 4. Resonance frequency as a function of the out-of-plane buckling of a $300 \mu \mathrm{m}$-long microbridge for the first six resonance modes.

\section{Influence of the dimensions of the microbridges}

To analyze the influence of the length and width of the microbridges on the frequency response, we have fabricated microbridges of two different sizes: $80 \mu \mathrm{m}$-wide by $300 \mu \mathrm{m}$-long and $100 \mu \mathrm{m}$-wide by $500 \mu \mathrm{m}$-long. Both sizes have been fabricated on the same chip; therefore, before been released, they had the same initial residual stress. The magnitude of the out-of-plane buckling changes with the length of the microbridge. It varies from $15 \mu \mathrm{m}$ for the $300 \mu \mathrm{m}$ - 
long bridge to around $30 \mu \mathrm{m}$ for the $500 \mu \mathrm{m}$-long. We have selected samples with high buckling (large initial stress) to make this comparison, although a similar behavior has been observed in microbridges with low buckling. Figure 5 shows the frequency response of two representative $300 \mu \mathrm{m}$-long (curve (a)) and $500 \mu \mathrm{m}$-long (curve (b)) microbridges.
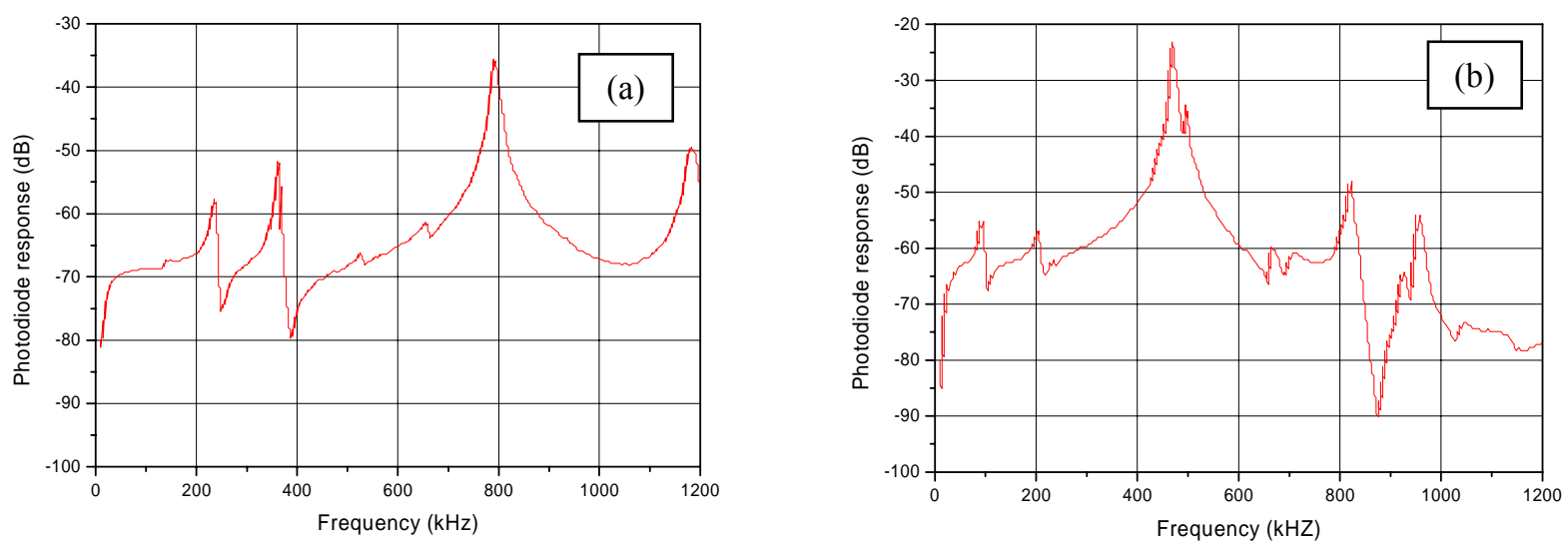

Figure 5. Frequency response of (a) a $300 \mu \mathrm{m}$-long microbridge and (b) a $500 \mu \mathrm{m}$-long microbridge.

The two spectra display the same set of resonance modes, their frequency being dependent on the size of the microbridge. The most intense mode appears at $795 \mathrm{kHz}$ for the $300 \mu \mathrm{m}$-long microbridge and at $470 \mathrm{kHz}$ for the 500 $\mu \mathrm{m}$-long. As expected, the rest of the modes are also shifted to lower frequencies for the $500 \mu \mathrm{m}$-long microbridge. These results show that it is possible to rely on the dimensions of the microbridges as another design parameter to adjust the operating frequency to the required specifications. For some applications such as chemical sensors, the effective surface of the resonator is a parameter that directly affects its performance. The detection limit of a gravimetric sensor (the smallest mass change that can be detected) increases with the resonance frequency. Besides, an increase of the sensing area allows to detect lower concentrations of the target analyte. A curved microbridge benefits from both effects, as it resonates at higher frequencies and provides a small increment of the sensing area that can enhance its performance for a given frequency of operation.

\section{Comparison between reflectometry and impedance measurements}

As we mentioned in the experimental section, the use of a piezoelectric capacitor to excite the microbridge allows to detect the vibrational movement by means of admittance measurements. Figure 6(a) shows the impedance of a $500 \mu \mathrm{m}$ long highly-buckled microbridge. The real part displays some peaks that correspond to resonance modes at $410 \mathrm{kHz}$ and $520 \mathrm{kHz}$. The imaginary part of the impedance is negative regardless of the resonance modes, a characteristic of the capacitive nature of the microbridge, The real part of the impedance, far enough from the peaks, varies slowly. Thus, out of resonance the microbridge behaves like a lossy capacitor. Figure 6(b) represents the spectrum obtained by laser interferometry. It shows the two resonances present in the impedance spectra plus some other at higher frequencies. 

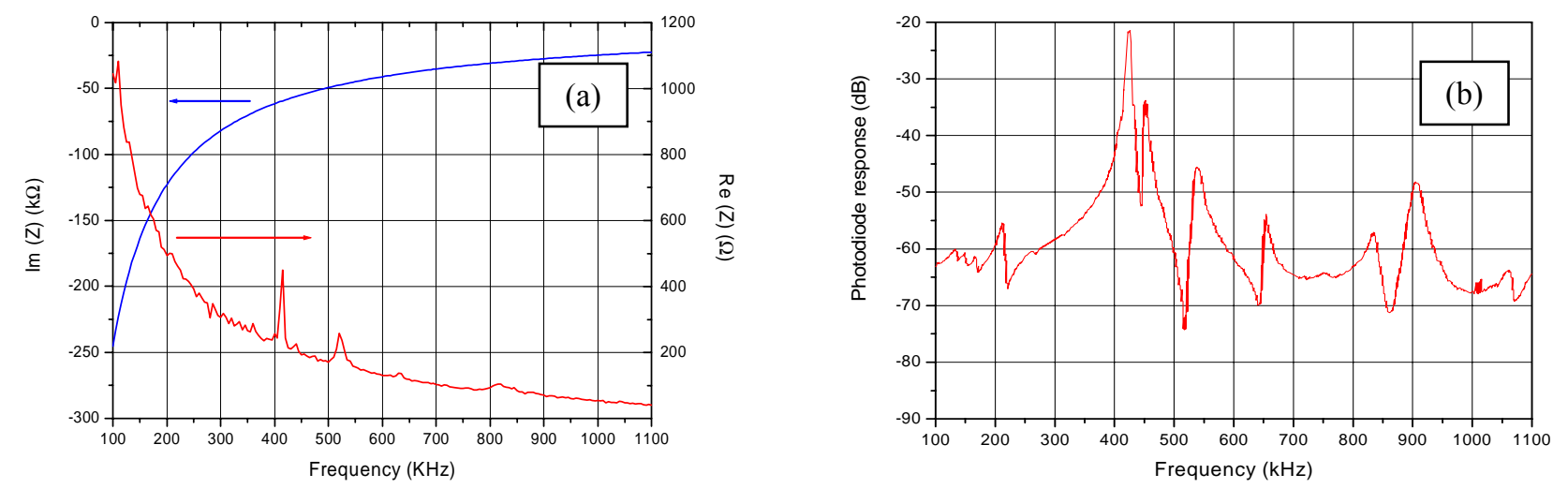

Figure 6. (a) Real and imaginary parts of the impedance of a $500 \mu \mathrm{m}$-long microbridge;

(b) frequency spectrum obtained by laser interferometry in the same microbridge.

To understand why fewer resonance modes appear in the impedance spectra compared to intereferometry spectra, it is necessary to consider what each technique is sensitive to. In both cases we have an electrical excitation consisting of a periodic voltage between the electrodes. Two different physical phenomena contribute to the overall reaction charge in a piezoelectric material such as AIN. One is related to the polarization of the material exposed to an external electric field. Since the AIN dielectric slab is surrounded by metallic electrodes, the associated impedance is mainly capacitive. On the other hand, additional reaction charges are produced due to the piezoelectric nature of the slab, also contributing to modify the polarization. These two contributions to the polarization can be expressed analytically (in one-dimensional notation) by:

$$
P=d_{33} T_{3}+\varepsilon_{\mathrm{o}} \chi E_{3}
$$

where $d_{33}$ is the piezoelectric strain constant, $T_{3}$ the stress, $\varepsilon_{\mathrm{o}}$ the permittivity of free space, $\chi$ the dielectric susceptibility and $E_{3}$ the electric field. The subindex 3 refers to the $c$-axis direction of the wurtzite crystal structure of AlN, perpendicular to the film surface. At resonance, the crystal lattice is subject to a rather pronounced strain; consequently, the surface reaction charge changes dramatically. The charge distribution is then directly related to the stress distribution and has similar symmetry. When the situation is such that the stress has both negative and positive contributions almost compensated, the overall reaction charge is negligible and the change in the impedance can not be detected. This occurs especially in modes with a strong torsional symmetry, where charges of equal magnitude and opposite sign are generated in symmetric parts of the electrodes, canceling each other out and giving negligible current (impedance). However, in the case of reflectometry, any resonance mode produces a variation of the intensity of the reflected laser beam as it detects the actual movements of the microbridge. This technique is independent of the charge or strain distribution over the microbridge.

\section{Fine-tuning of the resonance frequency by applying a DC bias}

As we reported previously, ${ }^{25}$ the application of a DC voltage to the microbridge causes a change of its buckling that can get as high as $3.6 \mu \mathrm{m}$ for a voltage of $16 \mathrm{~V}$. This additional buckling also affects the resonance frequency of the vibrational modes. Figure 7 shows the normalized spectra around a resonance mode of a $500 \mu \mathrm{m}$-long microbridge for three DC voltages added to the AC excitation signal. The DC bias induces a piezoelectric strain that changes the microbridge curvature, also affecting its resonance frequency. As the change in curvature is slightly different for positive or negative values of the DC bias, ${ }^{25}$ the frequency shift is also asymmetric. From the average shift it is deduced that the tuning factor of the microbridges is around $88 \mathrm{~Hz} / \mathrm{V}$. This value is high enough to induce variations of the operating frequency of the same order of magnitude as those expected during operation as chemical sensor, It is thus possible to conceive of sensing systems at constant-frequency. 


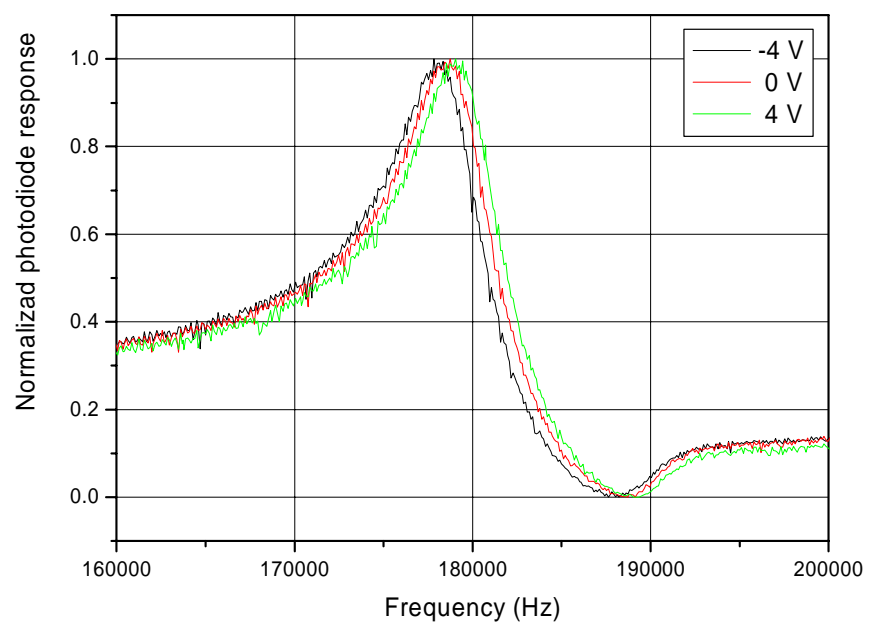

Figure 7. Normalized spectra of a $500 \mu \mathrm{m}$-long microbridge measured with three DC voltages added to the AC excitation signal.

\section{CONCLUSIONS}

We have studied the behavior of piezoelectrically-actuated AIN microbridges with potential use as microresonators for frequency filtering or gravimetric chemical sensing applications. The electromechanical response of the microbridges has been obtained by laser interferometry and electrical admittance measurements. Due to the residual stress of their constituent layers before releasing, microbridges became convexly curved after removing the sacrificial layer. This buckling increases significantly the resonance frequency of the different vibrational modes. This would allow one to pre-tune the resonance frequency during fabrication by controlling the residual stress of the structural layer. It is also possible to fine-tune the frequency after fabrication of the microbridge by applying a DC voltage added to the AC excitation signal. A tuning factor of around $88 \mathrm{~Hz} / \mathrm{V}$ has been obtained in a $500 \mu \mathrm{m}$-long microbridge. It is shown that impedance measurements provide a simple method to detect resonance frequencies, which show up as peaks in the real part of the impedance spectrum. This method takes advantage of the dual characteristic of the piezoelectric microbridges that can be excited and measured simultaneously through the same two electrodes.

\section{ACKNOWLEDGMENTS}

This work has been supported by the Ministerio de Educación y Ciencia of Spain through project no. MAT2004-00175 and by the Junta de Comunidades de Castilla-La Mancha through contract no. PAC05-001-2.

\section{REFERENCES}

1. H.-S. Lee, C. H. Leung, J. Shi, S-C. Chang, S. Lorincz, I. Nedelescu, "Integrated Microrelays: Concept and Initial Results", J. Microelectromech. Syst. 11(2) pp. 147-149, 2002.

2. E. Quandt, A. Ludwig, "Magnetostrictive actuation in Microsystems", Sensors and Actuators A 81 pp. 275-280, 2000. 
3. J.-Y. Lee, S.-W. Kang, "A characterization of the thermal parameters of thermally driven polysilicon microbridge actuators using electrical impedance analysis", Sensors and Actuators A 75 pp. 86-92, 1999.

4. E. Iborra, J. Olivares, M. Clement, L. Vergara, A. Sanz-Hervás, J. Sangrador, "Piezoelectric properties and residual stress of sputtered AIN thin films for MEMS applications", Sensors and Actuators A 115 pp. 501-507, 2004.

5. S. Pamidighantam, R. Puers, K. Baert, and H. Tilmans, "Pull-in voltage analysis of electrostatically actuated beam structures with fixed-fixed and fixed-free end conditions", J. Micromech. Microeng. 12 pp. 458-464, 2002.

6. J-L. A. Yeh, H. Jiang, and N. C. Tien, "Integrated polysilicon and drie bulk silicon micromachining for an electrostatic torsional actuator", J. Microelectromech. Syst. 8(4) pp. 456-465, 1999.

7. H. J. M. T. A. Adriaens, W. L. de Koning, and R. Banning, "Modelling Piezoelectric Actuators", IEEE/ASME Trans. Mechatronics 5(4) pp. 331-341, 2000.

8. M. Hoffmann, H. Küppers, T. Schneller, U. Böttger, U. Schnakenberg, W. Mokwa, and R. Waser, "Theoretical calculations and performance results of a PZT thin film actuator", IEEE Trans. Ultrason. Freq, Contr. 50(10) pp. 1240-1246, 2003.

9. T. Shibata, K. Unno, E. Makino, Y. Ito, S. Shimada, "Characterization of sputtered ZnO thin film as sensor and actuator for diamond AFM probe", Sensors and Actuators A 102 pp. 106-113, 2002.

10. L. J. Currano, A. E. Wickenden, M. Dubey, "Fabrication of fully released aluminum nitride nanoresonators", Proceedings of the 2003 third IEEE Conference on Nanotechnology. (IEEE-NANO 2003) 2 pp. 778-780, 2003.

11. A. Ballato, J. G. Gualtieri, J. A. Kosinski, "Ferroelectric materials for thin film and membrane resonators", Proc. Ninth IEEE Intl. Symp. on Aplications of Ferroelectrics, pp. 674, 1994.

12. B.H. Kim, D.P. Kern, S. Raible, U. Weimar, "Fabrication of micromechanical mass-sensitive resonators with increased mass resolution using SOI substrate", Microelectronic Engineering 61-62 pp. 947-953, 2002.

13. G. Piazza, R. Abdolvand, G. K. Ho, F. Ayazi, "Voltage-tunable piezoelectrically-transduced single-crystal silicon micromechanical resonators", Sensors and Actuators A 111 pp. 71-78, 2004.

14. H. Li, B. Balachandran, "Buckling and free oscillations of composite microresonators, J. Microelectromech. Syst. 15 pp. 42-51, 2006.

15. M. Zang, S. M. Zurn, D. L. Polla, B. J. Nelson, W. P. Robbins, "Design, simulation and fabrication of a bridge structure microtransducer", Technical Proceedings of the 2000 International Conference on Modelling and Simulation of Microsystems Chapter 6: Characterization, Parameter Extraction, Calibration pp. 265-268, 2000.

16. H. A. C. Tilmans, D. J. IJntema, J. H. J. Fluitman, "Single element excitation and detection of (micro-)mechanical resonators", 1991 International Conference on Solid-State Sensors and Actuators, Digest of Technical Papers, TRANSDUCERS'91 pp. 533-537, 1991.

17. S. Lee, R. Kazinczi, J. Mollinger, M. Vellekoop, A. Bossche, "FEM Study on the Dependence of Resonant Frequency Shift on Mechanical Stress of thin Film Resonator", Proceedings of Semiconductor Sensor and Actuator Technology, SeSens 2001 pp. 817-820, 2001.

18. Q.-M. Wang, T. Z., Q. Chen, X.-H. Du, "Effect of DC bias field on the complex materials coefficients of piezoelectric resonators", Sensors and Actuators A 109 pp. 149-155, 2003.

19. J. Olivares, E. Iborra, M. Clement, L. Vergara, J. Sangrador, A. Sanz-Hervás, "Piezoelectric actuation of microbridges using AlN", Sensors and Actuators A 123-124 pp. 590-595, 2005.

20. G. Meyer, N.M. Amer, "Novel optical approach to atomic force microscopy", Appl. Phys. Lett. 53 pp. 1045-1047 (1988).

21. J. Malo, J. Izpura, "Electronic driving of microcantilevers", submitted to Sensors and Actuators B.

22. Coventor Inc., CoventorWare ${ }^{\mathrm{TM}}$ Reference Guide, Cary, NC, 2002. www.coventor.com 
23. K. Tsubouchi, N. Mikoshiba, "Zero-temperature-coefficient SAW devices on AlN epitaxial films", IEEE trans. on Sonics and Ultrasonics SU-32 pp. 634-644, 1985.

24. www.matweb.com, searchable database of material properties, 2006.

25. J. Olivares, M. Clement, E. Iborra, L. Vergara, J. L. Sánchez-Rojas, L. M. Gutiérrez, J. Vázquez, "Simulation, Fabrication and Testing of Aluminium Nitride Piezoelectric Microbridges", Proceedings of the SPIE's International Symposium on Microtechnologies for the New Millenium 5836 pp 16-26, 2005. 